\title{
Psychological distress and resilience in patients with advanced cancer during the Covid-19 pandemic: the mediating role of spirituality
}

\section{Luka Mihic-Góngora}

Hospital Universitario Central de Asturias

Paula Jiménez-Fonseca

Hospital Universitario Central de Asturias

Raquel Hernández

Hospital Universitario de Canarias

Mireia Gil-Raga

Hospital General Universitario De Valencia

Vilma Pacheco-Barcia

Hospital Central de la Defensa Gómez Ulla

Aranzazu Manzano-Fernández

Hospital Clínico San Carlos

Susana Hernando-Polo

Hospital Universitario Fundación Alcorcón

Monica Antoñanzas-Basa

Hospital Clínico San Carlos

Maria J. Corral

University of Barcelona

María Valero-Arbizu

Hospital Quironsalud, Sevilla

Caterina Calderon ( $\nabla$ ccalderon@ub.edu )

University of Barcelona

\section{Research Article}

Keywords: advancer cancer, palliative care, disease outbreaks, psychological distress, resilience, end of life

Posted Date: March 3rd, 2022

DOI: https://doi.org/10.21203/rs.3.rs-1350855/v1 
License: (c) (i) This work is licensed under a Creative Commons Attribution 4.0 International License. Read Full License 


\section{Abstract}

Background. The purpose of this study was to investigate the sociodemographic factors related to psychological distress, spirituality, and resilience, and to examine the mediating role of spirituality with respect to psychological distress and resilience in patients with advanced, unresectable cancer during the Covid-19 pandemic.

Methods. A prospective, cross-sectional design was adopted. Data were collected from 636 participants with advanced cancer at 15 tertiary hospitals in Spain between February 2019 and December 2021. Participants completed self-report measures: Brief Resilient Coping Scale (BRCS), Brief Symptom Inventory (BSI-18), and Spiritual well-being (FACIT-Sp). Hierarchical linear regression models were used to explore the mediating role of spirituality.

Results. Spirituality was significantly different according to the person's age and marital status. Psychological distress accounted for $12 \%$ of the variance in resilience $(\beta=-0.32, p<0.001)$ and spirituality, another $15 \%(\beta=0.48, p<0.001)$. Spirituality acted as a partial mediator in the relationship between psychological distress and resilience in individuals with advanced cancer.

Conclusions. Both psychological distress and spirituality played a role in resilience in cases of advanced cancer. Spirituality can help promote subjective well-being and increased resilience in these subjects.

\section{Introduction}

Since the beginning of the coronavirus pandemic, more than 280 million cases of infection and more than five million deaths have been reported [1]. Immunosuppressed cancer patients are at greater risk of COVID-19 and demise related to it [2]. Fear of SARS-CoV-2 infection, difficulty in accessing medical care, fear of relapse or disease progression due to treatment delay or modification are more common concerns among individuals with cancer during this period shaped by the pandemic [3]. These apprehensions can be accompanied by greater psychological distress, increased anxiety and depression [4], causing them to be more preoccupied about dying, thereby aggravating their anguish and diminishing their quality of life [5].

Spirituality refers to how people search for and expression meaning and purpose in life and how they experience greater connection with themselves, others, and with the transcendental [6, 7]. According to Peterman et al. [6], spirituality can be understood as an essential element of health and overall well-being, and would therefore integrate dimensions of physical, psychological, and social health. It has attracted the attention of cancer research, inasmuch as it can help patients cope with the diagnostic and treatment processes better $[7,8]$, thereby contributing to reducing their psychological distress and enhancing their quality of life [8].

Resilience is another relevant aspect in the oncology population, as it can protect them from the detrimental effects of stress and adversity by softening the negative impact of the diagnosis; from 
treatment side effects, and from disease-related changes in lifestyle, and, in this way, improve their mental health and therapeutic outcomes $[9,10]$. In one study of individuals with cancer who underwent hematopoietic stem cells, the more resilient participants reported less psychological distress and better quality of life than the less resilient ones [11]. In contrast, less resilient subjects report more anguish and depression [12, 13], even long after treatment [12].

Both spirituality and resilience have much to do with a person's attempts to confront cancer and all the stressful events associated with it $[12,14]$. There are no studies that probe the mediating role of spirituality between psychological distress and resilience in people with metastatic cancer. Consequently, this study analyzes the relationship between sociodemographic data and spirituality, resilience, and psychological distress and the mediating function of spirituality between these variables in subjects with advanced, unresectable cancer during the COVID-19 pandemic COVID-19. We hypothesize that, in these cases, spirituality will be a determinant in the relationship between psychological distress and resilience.

\section{Methods}

\section{Design and patients}

This multi-institutional, prospective, observational study is part of a participant's research program funded by the Bioethics Group of the Spanish Society of Medical Oncology (SEOM). The study was conducted at 15 tertiary hospitals in Spain between February 2019 and December 2021, period coinciding with the COVID-19 pandemic. Participants aged 18 years and older with histologically confirmed advanced, unresectable cancer and candidates for systemic treatment were consecutively enrolled. Individuals with any serious mental illness that prevented survey comprehension were excluded. Eligible patients were invited to participate in the study during the first visit to the medical oncology department for systemic treatment. Those who agreed to participate signed the consent form, were given instructions on how to fill in the written questionnaires, completed at home and handed them to the auxiliary staff at the next visit. Information was collected from clinical records or directly from the participants by medical oncologists. The database is managed via an online platform (www.neoetic.es).

The study was performed in accordance with Good Clinical Practice guidelines and the Declaration of Helsinki. It was approved by research ethics board of each institution and classified by the Spanish Agency of Medicines and Medical Devices (AEMPS).

\section{Measures}

Participants completed the validated Spanish version of the following questionnaires.

The Brief Resilient Coping Scale (BRCS) is a 4-item [15], unidimensional outcome measure designed to capture what extent an individual cope with stress in a resilient fashion. The items have a response format with five options, where 1 means the statement "does not describe you at all" and 5 means "it describes you very well". The sum score varies between 4 to 20 , the higher the score, the more resilience. 
Cut-off values of $\leq 13$ and $\geq 17$ are used to differentiate between low and high resilience scores [15]. The internal consistency coefficient was 0.76 [15].

\section{Spiritual well-being was appraised by the Functional Assessment of Chronic Illness Therapy-Spiritual}

Well-Being Scale (FACIT-Sp) [6,16]. This instrument consists of 12 items scored on a five-point scale and contains two subscales, Meaning/Peace and Faith, and the total sum provide by the index of spiritual well-being. The higher the score, the greater the person's wellbeing. Reliability for scale ranged from 0.850.86 in the Spanish sample [17].

Brief Symptom Inventory 18 (BSI-18) consists of 18 items divided into three dimensions (somatizations, depression, and anxiety), and a total score, the Global Severity Index (GSI), which summarizes the respondent's overall emotional adjustment or psychological distress over the last 7 days [18]. Each item is rated on a 5 -point Likert scale from 0 (not at all) to 4 (extremely). Cronbach's alpha varied from 0.81 to 0.90 [18].

\section{Data analysis}

Data were statistically analyzed using the Statistical Package for Social Sciences (SPSS) for Windows 23.0 (SPSS Inc, Chicago, Illinois). All statistical tests were two-sided and the significance level was set at $p<0.05$. Descriptive statistics for demographic and other variables were indicated by mean, standard deviation (SD), number $(\mathrm{N})$ and percentage (\%) as appropriate. T-tests and one-way ANOVA were used to compare differences in spirituality, resilience, and psychological distress between categorical groups. Etasquared was reported as an indicator of the effect size of differences, with ranges between 0 and 1 , with $\eta^{2} \sim 01$ for a small, $\eta^{2} \sim 0.06$ for a medium and $\eta^{2}>0.14$ for a large effect size [19]. Pearson's correlation was used to examine correlations between continuous variables. Hierarchical regression analysis was used to explore the mediating effects of resilience on the relationship between psychological distress and life satisfaction. According to Baron and Kenny's technique on mediation [48], the following conditions should be met: (1) the independent variable (psychological distress) is significantly related to the dependent variable (resilience); (2) the independent variable (psychological distress) is significantly related to the mediator (spirituality); (3) the mediator (spirituality) is significantly related to the dependent variable (resilience), with the effect of the independent variable (psychological distress) on the dependent variable (resilience) upon adding the mediator (spirituality) to the model. Moreover, Sobel's test was performed to estimate the mediation effect.

\section{Results}

Of the 663 individuals recruited, 636 were eligible. A total of 27 were excluded ( 6 failed to meet the inclusion criteria; 5 met an exclusion criterion, and 16 had incomplete data). The mean age was 64.8 years (range, 24-89) and 53.1\% $(n=338)$ were male. Most were married $(84.4 \%)$, had a secondary education (50.8\%), and all were retired or unemployed (100\%). The most common cancers were bronchopulmonary (32.4\%), digestive (39.1\%), and breast (9.4\%). Adenocarcinoma histology was the 
most frequent (62.1\%) and most were stage IV (80.7\%). The most common treatment was chemotherapy (53.1\%), chemotherapy with targeted drug (14.2\%), and chemotherapy with immunotherapy $(12.1 \%)$. Estimated survival was less than 12 months in $26.3 \%$ of the sample. The characteristics of the study population can be found in Table 1.

Just over thirty percent (30.5\%) of the participants were found to be highly resilient copers. Patients $>70$ years and those with a primary education scored lower on resilience than those $\leq 70$ years and those with a higher education $\left(F=7.044, p=0.001, \eta^{2}=0.022 ; F=7.471, p=0.006, \eta^{2=} 0.012\right.$, respectively). Women displayed greater psychological distress than men $\left(F=14.985, p=0.001, \eta^{2=} 0.023\right)$, as did subjects $<50$ and $>70$ years of age $\left(F=4.775, p=0.009, \eta^{2=} 0.015\right)$. Participants $<50$ years and those without a partner scored the lowest on spirituality $\left(F=6.093, p=0.002, \eta^{2=0.019} ;=5.985, p=0.015, \eta^{2=} 0.012\right.$, respectively).

Insert Table 1 here

\section{Correlations across variables}

The mean, standard deviations of the variables, and Pearson correction analyses are presented in Table 2. The mean BRCS, BSI-18, and FACIT-Sp scores were $14.3 \pm 3.8,67.1 \pm 7.3$, and $36.5 \pm 6.6$, respectively. The results revealed that there were significant correlations across all psychological variables and that these correlations were in the direction expected. Psychological distress correlated negatively with resilience and spiritual well-being, while resilience correlated positively with spiritual well-being. Therefore, the first two conditions of Baron and Kenny's technique were met in the present study.

Insert Table 2 here

\section{The mediating role of resilience in the relationship between psychological distress and spiritual well-being}

Hierarchical linear regression analyses to explore the mediating role of spirituality are represented in Figure 1. After adjusting for sociodemographic factors, the results reveal that psychological distress was negatively associated with spirituality and resilience $(\beta=-0.32, p=0.001 ; \beta=-0.34, p=0.001$, respectively), whereas spirituality correlated positively with resilience $(\beta=0.48, p=0.001)$. Furthermore, spirituality mediated partially in the association between psychological distress and resilience, as the absolute value of its standardized regression coefficient ( $\beta$ ) decreased from -0.34 to -0.21 (Sobel test, $z=6.835, p=0.001$ ).

Insert Figure 1 here

\section{Discussion}

To the best of our knowledge, this is the first study to explore spiritual well-being in individuals with advanced cancer during the COVID-19 pandemic and to scrutinize the mediating role of spirituality between psychological distress and resilience in this population. The results established that spirituality played a $12.1 \%$ mediating role and, as expected, found a negative correlation between psychological distress, spirituality, and resilience. 
Many psychosocial studies have been conducted in patients with cancer in recent years [20]. Psychological distress and depression have been proven to be risk factors $[13,21]$ and spirituality to be protective in the development of depression [14, 22]. A systematic review of studies in cases of advanced cancer revealed that resilience is associated with spirituality, social support, the search for meaning, accepting their disease, positive attitude, and quality of life [12, 23]. During the COVID-19 pandemic, female breast cancer survivors who scored high on spirituality and resilience experienced less fear of recurrence, despite not receiving their usual medical follow-up [12, 23].

Despite the positive correlation between spirituality and resilience, they are considered to have their own, distinct characteristics $[22,24]$. Recent research points toward spirituality potentially increasing resilience in different ways: favoring interpersonal relationships, as a source of strength and inner solace, or deceasing feelings of anger and social isolation $[25,26]$. In this manner, spirituality could nurture resilience in patients with advanced cancer, but not vice versa, insofar as there can be resilient individuals without high levels of spirituality. This was found in the present study in which subjects $>70$ years were not the most resilient despite exhibiting higher levels of spirituality. The greater frailty and vulnerability (comorbidities, lower functionality) and dependency, and poorer tolerance to cancer treatment of the elderly may account for this finding. [27, 28]. Similarly, our results indicate that seniors ( $>70$ years) display less resilience and greater psychological distress. This is in line with earlier investigations that demonstrate that seniors have less resilience and more psychological issues like depression, attributable to their loneliness with less social support, lack of energy, and physical decline [29, 30].

Our study reveals that, graphically, psychological distress is U-shaped; i.e., it is highest in patients $\leq 50$ and $>70$ years. This could be due to young adults finding their chances of achieving their life goals limited by their diagnosis of advanced cancer [31]. In seniors, psychological distress has been associated with them being physically weaker and suffering greater psychological affliction given the loss of significant people in their surroundings [26]. Our study also displayed greater psychological distress among women than men, which is in keeping with the literature that point toward females with cancer being more prone to psychological problems and suffering more from the repercussions on their family and milieu, given the organic, cosmetic, functional, and cognitive sequelae following their cancer diagnosis and treatment, as well as presenting more sexual problems [32, 33].

The study has a series of strengths and limitations. First, while the COVID-19 outbreak was a fundamental motivator in this study, it was designed before then and none of the variables collected was associated with the pandemic nor were infected individuals included, given that they had to overcome the disease in order to attend their oncology appointment. Secondly, given its cross-sectional nature, we were unable to draw causal relations across study variables. The findings of the current study should be confirmed by longitudinal cohort studies in the future. Third, all data were obtained through self-report questionnaires, which could introduce response bias. The participants may have underestimated or overestimated the relationship between the study variables. Fourth, the study did not seek to nor was it statistically powered to compare behavior of patients with different neoplasms; hence, the weight of tumor type has not been analyzed in the findings. Finally, despite the fact that the sample is 
representative of the Spanish geography, any generalization of the results to other cultures and societies must be made with caution.

\section{Clinical implications}

People with advanced, unresectable cancer find their life expectancy shortened and confront a situation in which spiritual concerns arise. Spirituality can help in the face of end-of-life despair, endowing the situation and one's own existence with meaning and a sense of transcendence [12,22]. The importance of spirituality notwithstanding, it is not easy for physicians to talk about the spiritual concerns of patients with advanced cancer.

Spirituality-based coping mechanisms can help to promote subjective wellbeing and greater resilience in cases of incurable cancer. Resilience, underpinned by spirituality, can help in the process of adapting to the disease and at the end of life.

Regardless of society's secularization over the las 50 years, studies show that there is increased interest in spiritual growth and religious activity in older adults [22]. Including spirituality in interventions and the training of healthcare professionals who work with subjects with advanced cancer and in palliative care can contribute to maintaining and enhancing the resilience and wellbeing of patients and their caregivers $[34,35]$. Moreover, finding meaning to life, reformulating the narratives of loss, and being a member of a community, such as a religious community, are some ways in which spirituality can bolster resilience and help people handle the challenges of the disease. In conclusion, spirituality can help promote subjective wellbeing and resilience in individuals with advanced cancer.

\section{Declarations}

\section{Acknowledgements}

The authors grateful the investigators of the Neoetic study and the Bioetic Group of the Spanish Society of Medical Oncology (SEOM) for their contribution to this study. We would like to thank Priscilla Chase Duran for editing and translating the manuscript.

\section{Author's contributors}

LMG, PJF and CC contributed equally to this work and coordinated the project. They conducted the database search, screened, extracted the data, and wrote the first draft. All authors have made substantial contributions to the conception of the work, data collection, analysis, interpretation of data, and approved the final version to be published.

\section{Funding}

This study was funded by the FSEOM (Spanish Society of Medical Oncology Foundation) grant for Projects of the Collaborative Groups in 2018 and by an Astra Zeneca grant (ES2020-1939). The sponsor 
of this research has not participated in data collection, analysis, or interpretation; in writing the report, or in the decision to submit the article for publication.

\section{Data availability statement}

The datasets generated during and analyzed during the current study are not publicly available for reasons of privacy. They are however available (fully anonymised) from the corresponding author on reasonable request.

\section{Ethics approval and consent to participant}

The study was approved by the Research Ethics Committee of the Principality of Asturias (May 17, 2019) and by the Spanish Agency of Medicines and Medical Devices (AEMPS) (identification code: L34LMMM2GH-Y925U-RJDHQ). The study and all procedures have been performed in accordance with the ethical standards of the National Research Committee and the 1964 Declaration of Helsinki and its subsequent amendments. The study is an observational, non-interventionist trial. Informed consent was obtained from all participants.

\section{Consent for publication}

Not applicable.

\section{Competing interest}

The authors declare that they have no competing interest.

\section{References}

1. Worldmeter. COVID-19 Coronavirus Pandemic 2020. (accessed 18 Jan 2021).

2. Deng G, Yin M, Chen X, et al. Clinical determinants for fatality of 44,672 patients with COVID-19. Crit Care 2020;24:1-3.

3. Yang S, Dong D, Gu H, et al. Impact of stopping therapy during the SARS-CoV-2 pandemic in persons with lymphoma. J Cancer Res Clin Oncol 2020;147:1469-79. doi:10.1007/s00432-020-03426-0

4. Ayubi E, Bashirian S, Khazaei S. Depression and Anxiety Among Patients with Cancer During COVID19 Pandemic: A Systematic Review and Meta-analysis. J Gastrointest Cancer 2021;52:499-507. doi:10.1007/s12029-021-00643-9

5. Sigorski D, Sobczuk P, Osmola M, et al. Impact of COVID-19 on anxiety levels among patients with cancer actively treated with systemic therapy. ESMO Open 2020;5:1-8. doi:10.1136/esmoopen-2020000970

6. Peterman AH, Fitchett G, Brady MJ, et al. Measuring spiritual well-being in people with cancer: the functional assessment of chronic illness therapy-Spiritual Well-being Scale (FACIT-Sp). Ann Behav Med 2002;24:49-58. 
7. Damen A, Raijmakers NJH, van Roij J, et al. Spiritual Well-Being and Associated Factors in Dutch Patients With Advanced Cancer. J Pain Symptom Manage 2021;00:1-11.

doi:10.1016/j.jpainsymman.2021.10.004

8. Gayatri D, Efremov L, Kantelhardt EJ, et al. Quality of life of cancer patients at palliative care units in developing countries: systematic review of the published literature. Qual Life Res 2021;30:315-43. doi:10.1007/s11136-020-02633-z

9. Lau J, Khoo AM, Ho AH, et al. Psychological resilience among palliative patients with advanced cancer: A systematic review of definitions and associated factors. Psychooncology 2021;30:102940. doi:10.1002/pon.5666

10. Zhang J, Yizhen Y, Wang A, et al. Resilience in Patients With Lung Cancer. Cancer Nurs 2021;44:46572. doi:10.1097/NCC.0000000000000838

11. Andrykowski MA, Bishop MM, Hahn EA, et al. Long-Term Health-Related Quality of Life, Growth, and Spiritual Well-Being After Hematopoietic Stem-Cell Transplantation. 2011;23:599-608. doi:10.1200/JC0.2005.03.189

12. Koral L, Cirak Y. The relationships between fear of cancer recurrence, spiritual well-being and psychological resilience in non-metastatic breast cancer survivors during the COVID-19 outbreak. Psychooncology 2021;30:1765-72. doi:10.1002/pon.5727

13. Fradelos EC, Papathanasiou I V, Veneti A, et al. Psychological Distress and Resilience in Women Diagnosed with Breast Cancer in Greece. Asian Pac J Cancer Prev 2017;18:2545-50. doi:10.22034/APJCP.2017.18.9.2545

14. Çakir H, Çelik GK, Çirpan R. Correlation between social support and psychological resilience levels in patients undergoing colorectal cancer surgery: a descriptive study. Psychol Health Med 2021;26:899-910. doi:10.1080/13548506.2020.1859561

15. Sinclair VG, Wallston KA. The development and psychometric evaluation of the Brief Resilient Coping Scale. Assessment 2004;11:94-101.

16. Jimenez-Fonseca $P$, Calderon C, Carmona-Bayonas A, et al. The relationship between physician and cancer patient when initiating adjuvant treatment and its association with sociodemographic and clinical variables. Clin Transl Oncol 2018;:1-8. doi:10.1007/s12094-018-1870-z

17. Jimenez-Fonseca P, Lorenzo-Seva U, Ferrando PJ, et al. The mediating role of spirituality (meaning, peace, faith) between psychological distress and mental adjustment in cancer patients. Support Care Cancer 2018;26:1411-8. doi:10.1007/s00520-017-3969-0

18. Derogatis LR. BSI 18, Brief Symptom Inventory 18: Administration, scoring and procedures manual. Minneapolis: NCS Pearson, Inc. 2001.

19. Pierce CA, Block RA, Aguinis $\mathrm{H}$. Cautionary note on reporting eta-squared values from multifactor ANOVA designs. Educ Psychol Meas 2004;64:916-24.

20. Lau J, Ming A, Khoo G, et al. Resiliencia psicológica entre pacientes paliativos con cáncer avanzado: una revisión sistemática de definiciones y factores asociados. Psychooncology 2021;30:1029-40. doi:10.1002/pon.5666 
21. Zainal NZ, Nik-Jaafar NR, Baharudin A, et al. Prevalence of depression in breast cancer survivors: a systematic review of observational studies. Asian Pac J Cancer Prev 2013;14:264956.http://www.ncbi.nlm.nih.gov/pubmed/23725190

22. Manning L, Ferris M, Narvaez Rosario C, et al. Spiritual resilience: Understanding the protection and promotion of well-being in the later life. J Relig Spiritual Aging 2019;31:168-86. doi:10.1080/15528030.2018.1532859

23. Lau J, Ming A, Khoo G, et al. Psychological resilience among palliative patients with advanced cancer: A systematic review of definitions and associated factors. Psychooncology 2021;30:102940. doi:10.1002/pon.5666

24. Schwalm F, Zandavalli R, Castro-Filho E, et al. systematic review and meta-analysis of observational studies Is there a relationship between spirituality / religiosity and resilience ? A systematic review and meta- analysis of observational studies. J Health Psychol 2021;3:1-15. doi:10.1177/1359105320984537

25. Park Y, Suh S, Kim S, et al. Tag edH1 Development of a One-item Screening Question to Assess Spiritual Well-Being for Advanced Cancer Inpatients in Korea Tag edEn Tag edP Abstract. J Pain Symptom Manage 2021;62:910-7. doi:10.1016/j.jpainsymman.2021.05.005

26. Tamura S, Suzuki K, Ito Y, et al. Factors related to the resilience and mental health of adult cancer patients: a systematic review. Support Care Cancer 2021;29:3471-86. doi:10.1007/s00520-02005943-7

27. Santos FN, Castria TB De. Chemotherapy for advanced non-small cell lung cancer in the elderly population. 2016;134:465-6. doi:10.1590/1516-3180.20161345T1

28. Hernández R, Calderon C, Carmona-Bayonas A, et al. Differences in coping strategies among young adults and the elderly with cancer. Psychogeriatrics 2019;19. doi:10.1111/psyg.12420

29. Kocalevent R, Zenger M, Hinz A, et al. Resilient coping in the general population: standardization of the brief resilient coping scale (BRCS). Health Qual Life Outcomes 2017;15:251. doi:10.1186/s12955-017-0822-6

30. Castillo; RD, González Escobar, Sergio; Ivonne González Arratia López Fuentes N, Montero-López Lena M. Resiliencia en adultos mayores: estudio de revisión. Neurma Rev Electron Psicogerontologia 2017;4:22-9.

31. Burgers VWG, van der Graaf WTA, van der Meer DJ, et al. Adolescents and Young Adults Living With an Uncertain or Poor Cancer Prognosis: The “New” Lost Tribe. J Natl Compr Cancer Netw 2021;19:240-6. doi:10.6004/jnccn.2020.7696

32. Koyama A, Matsuoka H, Ohtake Y, et al. Gender differences in cancer-related distress in Japan: a retrospective observation study. Biopsychosoc Med 2016;:1-8. doi:10.1186/s13030-016-0062-8

33. Ghanem I, Castelo B, Jimenez-Fonseca P, et al. Coping strategies and depressive symptoms in cancer patients. Clin Transl Oncol 2020;22:330-6.

34. Hau A, Ho Y, Tan-ho G, et al. A novel mindful-compassion art therapy (MCAT) for reducing burnout and promoting resilience for end-of-life care professionals: a waitlist RCT protocol. Trials 2019;20:1- 
10.

35. Choo PY, Tan-Ho G, Dutta O, et al. Reciprocal Dynamics of Dignity in End-of-Life Care: A Multiperspective Systematic Review of Qualitative and Mixed Methods Research. Am J Hosp Palliat Med 2020;37:385-98. doi:10.1177/1049909119878860

\section{Tables}

Table 1. Comparison of mean total scores for psychological resilience (BRCS), psychological distress (BSI), and spiritual well-being (FACIT) according to baseline sample characteristics.

\begin{tabular}{|c|c|c|c|c|}
\hline Characteristics & $N(\%)$ & $\begin{array}{l}\text { BRCS } \\
(\text { mean } \pm S D)\end{array}$ & $\begin{array}{l}\text { BSI-18 } \\
(\text { mean } \pm \text { SD) }\end{array}$ & $\begin{array}{l}\text { FACIT-Sp } \\
(\text { mean } \pm S D)\end{array}$ \\
\hline \multicolumn{5}{|l|}{ Sex } \\
\hline Male & $338(53.1)$ & $14.5 \pm 3.9$ & $66.1 \pm 7.1$ & $36.5 \pm 6.8$ \\
\hline Female & $298(46.9)$ & $14.0 \pm 3.8$ & $68.3 \pm 7.3$ & $36.4 \pm 6.4$ \\
\hline$p$ value & & 0.080 & 0.001 & 0.719 \\
\hline \multicolumn{5}{|l|}{ Age } \\
\hline$\leq 50$ years & $56(8.8)$ & $14.6 \pm 3.2$ & $69.8 \pm 7.4$ & $33.6 \pm 6.7$ \\
\hline $51-70$ years & $392(61.6)$ & $14.6 \pm 3.7$ & $66.6 \pm 7.3$ & $36.6 \pm 6.5$ \\
\hline$>70$ years & $188(29.5)$ & $13.4 \pm 4.2$ & $67.4 \pm 7.3$ & $37.4 \pm 6.6$ \\
\hline$p$ value & & 0.001 & 0.009 & 0.002 \\
\hline \multicolumn{5}{|l|}{ Marital Status } \\
\hline Married/ partnered & $489(76.8)$ & $14.5 \pm 3.8$ & $66.8 \pm 7.3$ & $36.9 \pm 6.3$ \\
\hline Not partnered & $147(23.1)$ & $18.8 \pm 3.2$ & $68.2 \pm 6.9$ & $34.9 \pm 6.9$ \\
\hline$p$ value & & 0.447 & 0.120 & 0.015 \\
\hline \multicolumn{5}{|l|}{ Educational level } \\
\hline Primary & $313(49.2)$ & $13.8 \pm 4.1$ & $67.6 \pm 7.3$ & $37.0 \pm 6.6$ \\
\hline High school or higher & $323(50.8)$ & $14.7 \pm 3.6$ & $66.7 \pm 7.3$ & $36.0 \pm 6.7$ \\
\hline$p$ value & & 0.006 & 0.115 & 0.061 \\
\hline \multicolumn{5}{|c|}{$\begin{array}{l}\text { Abbreviations: BRCS, Brief Resilience Scale; BSI-18, Brief Symptom Inventory; FACIT, Functional } \\
\text { Assessment of Chronic Illness Therapy-Spiritual Well-Being Scale. }\end{array}$} \\
\hline Bold values indicate th & cant at $5 \%$ & & & \\
\hline
\end{tabular}


Table 2. Correlations between BRCS, BSI, and FACIT-Sp scores.

\begin{tabular}{lllll} 
& Mean & SD & BRCS & BSI-18 \\
\hline BRCS & 14.3 & 3.8 & 1 & \\
\hline BSI-18 & 67.1 & 7.3 & $-0.348^{\star \star}$ & 1 \\
\hline FACIT-Sp & 36.5 & 6.6 & $0.482^{\star \star}$ & $-0.320 * \star$
\end{tabular}

Abbreviations: SD, standard deviation: BRCS, Brief Resilience Scale; BSI-18, Brief Symptom Inventory; FACIT-Sp, Functional Assessment of Chronic Illness Therapy-Spiritual Well-Being Scale.

$\star \star p<0.001$

\section{Figures}

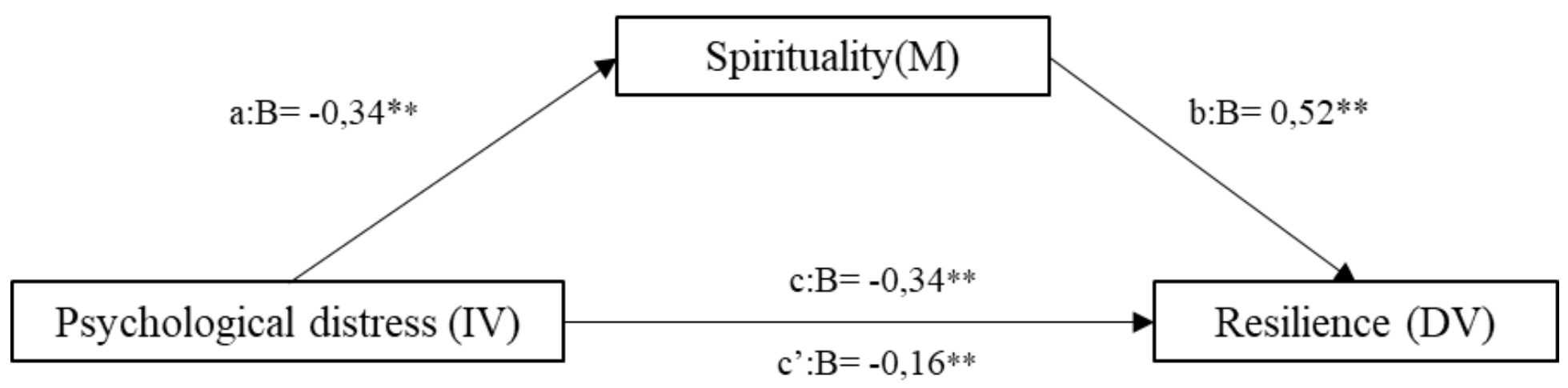

Figure 1

Mediation model for psychological distress, spirituality, and resilience.

$a=$ direct effect of independent variable (IV) on mediator (M). $b=$ direct effect of mediator on dependent variable (DV). c=direct effect of IV on DV. $c^{\prime}=$ indirect effect of IV on DV.

${ }^{\star} p<0.05 .{ }^{* \star} p<0.01$. 